\title{
Multiobjective Optimization of Low-Specific-Speed Multistage Pumps by Using Matrix Analysis and CFD Method
}

\author{
Qiaorui Si, Shouqi Yuan, Jianping Yuan, Chuan Wang, and Weigang Lu \\ Research Center of Fluid Machinery Engineering and Technology, Jiangsu University, Zhen Jiang 212013, China \\ Correspondence should be addressed to Qiaorui Si; siqiaoruijsu@163.com
}

Received 16 June 2013; Revised 22 August 2013; Accepted 22 August 2013

Academic Editor: Mohamed Fathy El-Amin

Copyright ( 2013 Qiaorui Si et al. This is an open access article distributed under the Creative Commons Attribution License, which permits unrestricted use, distribution, and reproduction in any medium, provided the original work is properly cited.

\begin{abstract}
The implementation of energy-saving and emission-reduction techniques has become a worldwide consensus. Thus, special attention should be provided to the field of pump optimization. With the objective of focusing on multiobjective optimization problems in low-specific-speed pumps, 10 parameters were carefully selected in this study for an $L_{27}\left(3^{10}\right)$ orthogonal experiment. The parameters include the outlet width of the impeller blade, blade number, and inlet setting angle of the guide vane. The numerical calculation appropriate for forecasting the performance of multistage pumps, such as the head, efficiency, and shaft power, was analyzed. Results were obtained after calculating the two-stage flow field of the pump through computational fluid dynamics (CFD) methods. A matrix method was proposed to optimize the results of the orthographic experiment. The optimal plan was selected according to the weight of each factor. Calculated results indicate that the inlet setting angle of the guide vane influences efficiency significantly and that the outlet angle of blades has an effect on the head and shaft power. A prototype was produced with the optimal plan for testing. The efficiency rating of the prototype reached 58.61\%; maximum shaft power was within the design requirements, which verifies that the proposed method is feasible for pump optimization.
\end{abstract}

\section{Introduction}

Pumps are widely utilized in various fields of the national economy [1]. Where there is water, there are pumps. Lowspecific-speed centrifugal pumps are commonly employed in sewage treatment and well pumping because of their small flow and high head. They consume large amounts of energy and have a potential for energy saving. Low efficiency and overload tendency in long-term large-flow operations are the two obstacles in designing such pumps; the impeller diameter of low-specific-speed centrifugal pumps is relatively large, and the flow channel is long and narrow, resulting in significant disc friction and hydraulic loss. Thus, the efficiency of low-specific-speed centrifugal pumps is relatively low; the shaft power curves of such pumps increase sharply. The greater flow method is usually adopted in the design of lowspecific-speed pumps to enhance flow efficiency. Thus, the ratio of macroshaft power to designed power becomes much higher than that of common centrifugal pumps. A motor whose export pipeline has no valve burns out easily $[2,3]$. Pumps are usually designed in multistages to increase the amount of pressure because of limitations imposed by costs or diameter, such as the case of well pumps. Increasing the single-stage head may decrease the number of stages to save costs, energy, and materials. High efficiency in maintaining the head and low power are the two goals of multistage pump designs. Thus, the study on multitarget optimal pump designs is important in implementing energy saving and emission reduction.

Mathematical methods, such as neural networks, orthographic experiments, genetic algorithms, and grey theory, are often adopted in multitarget optimization design $[4,5]$. An orthographic experiment design can optimize the experimental conditions to achieve the target with fewer experiments $[6,7]$. It has been widely adopted in pump design. Shouqi et al. [8] employed an orthogonal table $L_{8}\left(2^{7}\right)$ to thoroughly study the effect of impeller geometric parameters and the throat area on pump performance. He proposed a practical framework for a centrifugal pump without overload and its design method. Wang et al. [9] focused on the effect of main impeller geometric parameters on deep-geometric performance via orthographic experiments. Shen et al. [10] designed nine models of complex impeller centrifugal pumps with orthographic experiments and discovered the effect 


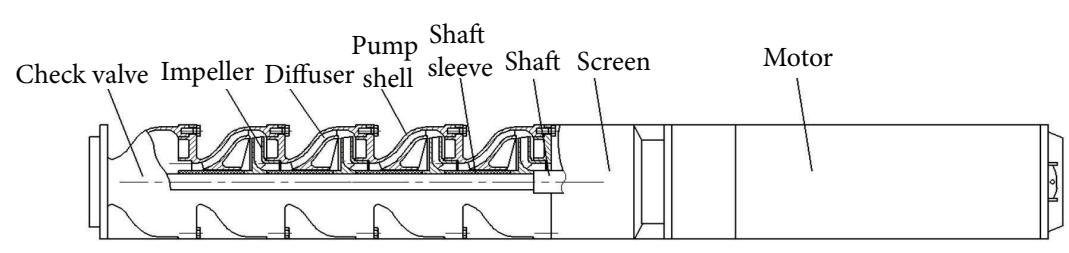

FIGURE 1: Model structure chart.

TABLE 1: Data structure of the multi-target orthogonal test.

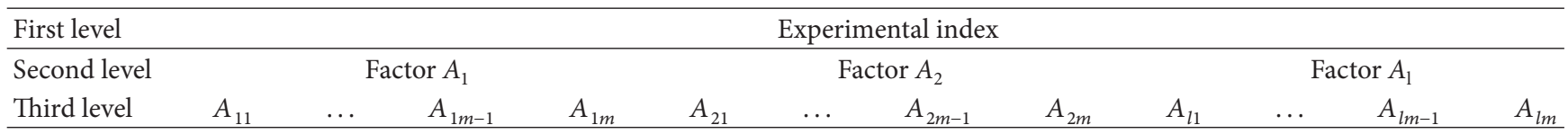

order of geometric parameters on pump performance. Zhou et al. [11] identified the key factors that affect the performance of guiding blades by studying conduit guiding blades and by conducting orthographic experiments and designing highperformance guiding blades. The orthogonal experiment method is therefore a multivariate and multilevel optimization method suitable for pump design. The studies mentioned above examined the influence of parameters on the impeller or guiding blades separately; however, the factors were not enough to completely reflect the effect of geometric parameters on the experiment. Furthermore, the multiobjective test was usually transformed into a single-objective test in the experimental analysis. Plans were then comprehensively selected through single-index analyses, including intuitive analysis and variance analysis. These methods neglect the significance and differences of indexes.

QS10-68, a typical multistage electric submersible pump (ESP), was regarded as an example in the present study. A method that combines orthogonal experimentation and numerical simulation was applied for optimization. A simple matrix method was also introduced to calculate the effect of factors on each index and to directly decide the factor order according to weight, which would perfectly solve the issue of selecting an optimal plan in an orthographic experiment design. The effect of 10 geometric parameters of the impeller and guiding vane on the multistage head, efficiency, and shaft power was also studied. Lastly, a set of optimum geometric parameters was obtained based on weight calculation. A prototype that employs the optimal plan was then tested for verification.

\section{Pump Model}

The structure of the multistage ESP is displayed in Figure 1. The figure shows a multistage centrifugal pump operating in a vertical position [12]. The pump shaft is connected to the protector by a mechanical coupling at the bottom of the pump. Fluids from the well enter the pump through an intake screen and are lifted by the pump stages. Produced liquids, after being subjected to great centrifugal forces caused by the high rotational speed of the impeller, lose their kinetic energy in the diffuser. Kinetic energy is converted to pressure energy in the diffuser. The design parameters for this pump are as follows: flow rate $Q_{d}=10 \mathrm{~m}^{3} / \mathrm{h}$, total head $H_{d}=68 \mathrm{~m}$, speed $n=2850 \mathrm{r} / \mathrm{min}$, four stages, and the efficiency of the necessary electric motor is $4 \mathrm{~kW}$.

\section{Matrix Analysis Model and Experimental Scheme}

In a multiobjective optimization problem, engineers adopt an orthographic experimental method as a solvent. The orthographic experimental method is a scientific method utilized to arrange and analyze multiple factor experiments by means of a table based on orthogonal principles. Through scientific arrangement and analysis of the result, a study can discover the ideal production conditions and techniques. However, problems, including large calculated amounts and confirming the weights unreasonably, exist in the multitarget orthogonal test method. A matrix analysis model was presented to solve this problem. In this model, a three-layer structure and the layer structure matrix of the orthogonal test are established first. The weight matrix of the test index is then calculated by multiplying the matrix of each layer. The weighs of the factors and levels that affect the tests results are calculated. Finally, the optimal plan and the importance order of the factors that influence the test index values are determined according to the weights.

3.1. Matrix Analysis Model. An orthographic experiment was designed (ignoring the interaction for the first). The three-layer structural model based on data structure was established. The first layer in Table 1 is the investigation index, the second layer is the factor layer, and the last one is the level layer. The data of each layer determine the definition of the matrix as follows.

Definition 1. The following is the matrix of the experimental investigation index. The study order is $K_{i j}=k_{i j}$. The matrix is established on the conditions that $l$ factors exist in the experiment, each factor has $m$ levels, $k_{i j}$ is the average index of factor $A_{i}$ at $j$ level, and the investigation index is good when it is high. A low investigation index is considered good when the study establishes a matrix supposing $K_{i j}=1 / k_{i j}$.

Definition 2. The matrix of the factor layer: $T_{i}=1 / \sum_{j=1}^{m} K_{i j}$ to build (2) matrix. 
Definition 3. The matrix of the level layer: the range of $A_{i}$ in the orthographic experiment is set as $s_{i}$. The study order is $S_{i}=s_{i} / \sum_{i=1}^{l} s_{i}$. Equation (3) matrix is then established.

Definition 4. The weight matrix that may affect the test index: $\omega^{T}=$ MTS.

$$
\begin{gathered}
M=\left[\begin{array}{ccccc}
K_{11} & 0 & 0 & \cdots & 0 \\
K_{12} & 0 & 0 & \cdots & 0 \\
\cdots & \ldots & \cdots & \cdots & \cdots \\
K_{1 m} & 0 & 0 & \cdots & 0 \\
0 & K_{21} & 0 & \cdots & 0 \\
0 & K_{22} & 0 & \cdots & 0 \\
\cdots & \cdots & \cdots & \cdots & \cdots \\
0 & K_{2 m} & & \cdots & \\
\cdots & \cdots & \ldots & \cdots & \cdots \\
0 & 0 & 0 & \cdots & K_{l 1} \\
0 & 0 & 0 & \cdots & K_{l 2} \\
\cdots & \cdots & \cdots & \cdots & \cdots \\
0 & 0 & 0 & \cdots & K_{l m}
\end{array}\right], \\
T=\left[\begin{array}{cccc}
T_{1} & 0 & 0 & 0 \\
0 & T_{2} & 0 & 0 \\
\cdots & \cdots & \cdots & \cdots \\
0 & 0 & 0 & T_{l}
\end{array}\right], \\
\omega^{T}=\left[\begin{array}{c}
\omega_{1}, \omega_{2}, \ldots, \omega_{m}
\end{array}\right] . \\
S=\left[\begin{array}{c}
S_{2} \\
\cdots \\
S_{l}
\end{array}\right],
\end{gathered}
$$

In the matrix above mentioned, $\omega_{1}=K_{11} T_{1} S_{1}, K_{11} T_{1}$ refers to the ratio of $A_{1}$ 's first level index to the sum of all $A_{1}$ 's level indexes. $S_{1}$ is the ratio of $A_{1}$ 's range to the sum of all ranges. The product of these two data reflects the effect of the first level of $A_{1}$ on the index and range of $A_{1}$. The other factors and levels are identical. The weight of each factor and level was obtained after calculation. The optimal plan and the factor order in the index were established based on the weight value.

3.2. Experimental Scheme. According to Euler equations, theoretical head $H_{t}$ is

$$
H_{t}=\frac{u_{2} v_{u 2}-u_{1} v_{u 1}}{g}=\frac{u_{2}}{g}\left(u_{2} h_{0}-\frac{Q_{t}}{F_{2} \tan \beta_{2}}\right),
$$

where $u_{1}(\mathrm{~m} / \mathrm{s})$ is peripheral speed at the inlet; $v_{u 1}$ is the peripheral velocity component of the blade inlet; $v_{u 1}=0$ in the straight cone suction chamber; $u_{2}(\mathrm{~m} / \mathrm{s})$ refers to the circular velocity at the impeller outlet; $g\left(\mathrm{~m}^{2} / \mathrm{s}\right)$ is acceleration because of gravity; $h_{0}=1-(\pi / z)$ is the stodala slip coefficient; $z$ represents blade numbers; $Q_{t}\left(\mathrm{~m}^{3} / \mathrm{h}\right)$ is the theoretical flow; $F_{2}=\pi D_{2} b_{2} \psi_{2}$ is the cross-section area of the impeller outer; $\psi_{2}$ is the blade expelling coefficient; $b_{2}(\mathrm{~m})$ is the width of the blade outlet; $\beta_{2}$ is the blade outlet angle. $P^{\prime}$, the hydraulic

\begin{tabular}{|c|c|c|c|c|c|c|c|c|c|c|}
\hline \multirow{2}{*}{ Level } & \multicolumn{10}{|c|}{ Factor } \\
\hline & $A$ & $B$ & $C$ & $D$ & $E$ & $F$ & $G$ & $H$ & $I$ & $J$ \\
\hline 1 & 122 & 8 & 10 & 6 & 2 & 88 & 34 & 5 & 0 & 15 \\
\hline 2 & 124 & 9 & 15 & 7 & 3 & 89 & 36 & 10 & 5 & 20 \\
\hline 3 & 126 & 10 & 20 & 8 & 4 & 90 & 38 & 15 & 10 & 25 \\
\hline
\end{tabular}
power input, can be deduced by the following equation:

$$
P^{\prime}=\rho g H_{t} Q_{t}=\rho u_{2}^{2} Q_{t}\left(h_{0}-\frac{Q_{t}}{u_{2} F_{2} \tan \beta_{2}}\right) \text {. }
$$

TABLE 2: Factor level table.

In (6), $\rho\left(\mathrm{kg} / \mathrm{m}^{3}\right)$ refers to water density. The equation shows that both theoretical head and hydraulic input power are determined by the impeller. However, rolling flow causes $P_{m}$, additional shaft power, as it enters the guiding blade channel. Hence, shaft power $P=P^{\prime}+P_{m}$ and pump efficiency $\eta=P^{\prime} / P$. Pump shaft power and efficiency are determined by both the impeller and the guide vane in hydraulics.

The following are the main geometric parameters of the model: width of the blade outlet $b_{2}$, suction diameter of impeller $d_{1}$, inclination of the back cover board $\alpha_{2}$, inlet attack angle $\Delta \beta_{1}$, inlet angle in axial plane $\gamma_{3}$, blade outlet angle $\beta_{2}$, blade number $z$, outer diameter of the back cover $d_{2 \text { min }}$, blade thickness $S_{2}$, inlet angle of the guide vane $\beta_{3}$, outlet angle of the guide vane $\alpha_{3}$, import width of the guide vane $b_{3}$, blade number of the guide vane $z_{1}$, axial length of the guide vane $\gamma_{3}$, inlet diameter of the guide vane $D_{2}$, and outlet diameter of the guide vane $D_{1} . \alpha_{3}$ is equal to $90^{\circ}$ to eliminate the rotation component. The last five factors were determined by the basic geometric parameters of the impeller. The first 10 geometric parameters were selected as testing factors.

According to the specific speed equation,

$$
n_{s}=\frac{3.65 n \sqrt{Q}}{H^{3 / 4}}
$$

where $Q$ refers to the flow and $H$ refers to the head of the pump. The design-specific speed of the pump is low, namely, 65.6, because it is lower than 80 . Relative parameters can be obtained with the velocity-coefficient method under structure demand; these factors are shown in Table 2. Three levels were selected in each factor. The experimental scheme, shown in Table 3 , is based on the $L_{27}\left(3^{10}\right)$ orthogonal table. In the 27 group tests, different numerals appear at the same frequency in any column, and any two-digit number appears equivalent in any row. After deciding the experimental plan, tests were implemented in numerical order without any arbitrary changes.

\section{Numerical Simulation}

In traditional orthogonal experiments, research procedures involve manufacturing prototypes, performance tests, and results analyses. However, creating prototypes from the 27 groups of impellers and guide vanes would be a waste of time and money; moreover, a large number of prototype tests would inevitably cause significant manufacturing and test errors. The prediction of pump performance has become 
TABLE 3: Orthogonal test schemes.

\begin{tabular}{|c|c|c|c|c|c|c|c|c|c|c|}
\hline Test number & $A / \mathrm{mm}$ & $B / \mathrm{mm}$ & $C /\left(^{\circ}\right)$ & $D /$ & $E / \mathrm{mm}$ & $F /\left(^{\circ}\right)$ & $\mathrm{G} / \mathrm{mm}$ & $H /\left(^{\circ}\right)$ & $I /\left(^{\circ}\right)$ & $J /\left(^{\circ}\right)$ \\
\hline 1 & 122 & 8 & 10 & 6 & 2 & 88 & 34 & 5 & 0 & 15 \\
\hline 2 & 122 & 8 & 10 & 6 & 3 & 89 & 36 & 10 & 5 & 20 \\
\hline 3 & 122 & 8 & 10 & 6 & 4 & 90 & 38 & 15 & 10 & 25 \\
\hline 4 & 122 & 9 & 15 & 7 & 2 & 88 & 34 & 10 & 5 & 20 \\
\hline 5 & 122 & 9 & 15 & 7 & 3 & 89 & 36 & 15 & 10 & 25 \\
\hline 6 & 122 & 9 & 15 & 7 & 4 & 90 & 38 & 5 & 0 & 15 \\
\hline 7 & 122 & 10 & 20 & 8 & 2 & 88 & 34 & 15 & 10 & 25 \\
\hline 8 & 122 & 10 & 20 & 8 & 3 & 89 & 36 & 5 & 0 & 15 \\
\hline 9 & 122 & 10 & 20 & 8 & 4 & 90 & 38 & 10 & 5 & 20 \\
\hline 10 & 124 & 8 & 15 & 8 & 2 & 89 & 38 & 5 & 5 & 25 \\
\hline 11 & 124 & 8 & 15 & 8 & 3 & 90 & 34 & 10 & 10 & 15 \\
\hline 12 & 124 & 8 & 15 & 8 & 4 & 88 & 36 & 15 & 0 & 20 \\
\hline 13 & 124 & 9 & 20 & 6 & 2 & 89 & 38 & 10 & 10 & 15 \\
\hline 14 & 124 & 9 & 20 & 6 & 3 & 90 & 34 & 15 & 0 & 20 \\
\hline 15 & 124 & 9 & 20 & 6 & 4 & 88 & 36 & 5 & 5 & 25 \\
\hline 16 & 124 & 10 & 10 & 7 & 2 & 89 & 38 & 15 & 0 & 20 \\
\hline 17 & 124 & 10 & 10 & 7 & 3 & 90 & 34 & 5 & 5 & 25 \\
\hline 18 & 124 & 10 & 10 & 7 & 4 & 88 & 36 & 10 & 10 & 15 \\
\hline 19 & 126 & 8 & 20 & 7 & 2 & 90 & 36 & 5 & 10 & 20 \\
\hline 20 & 126 & 8 & 20 & 7 & 3 & 88 & 38 & 10 & 0 & 25 \\
\hline 21 & 126 & 8 & 20 & 7 & 4 & 89 & 34 & 15 & 5 & 15 \\
\hline 22 & 126 & 9 & 10 & 8 & 2 & 90 & 36 & 10 & 0 & 25 \\
\hline 23 & 126 & 9 & 10 & 8 & 3 & 88 & 38 & 15 & 5 & 15 \\
\hline 24 & 126 & 9 & 10 & 8 & 4 & 89 & 34 & 5 & 10 & 20 \\
\hline 25 & 126 & 10 & 15 & 6 & 2 & 90 & 36 & 15 & 5 & 15 \\
\hline 26 & 126 & 10 & 15 & 6 & 3 & 88 & 38 & 5 & 10 & 20 \\
\hline 27 & 126 & 10 & 15 & 6 & 4 & 89 & 34 & 10 & 0 & 25 \\
\hline
\end{tabular}

possible in engineering applications owing to the rapid development of computational fluid dynamics (CFD) [13]. Reasonable CFD calculations reflect the actual internal flow of the pump and accurately predict the pump head performance, efficiency, shaft power, and so forth at specific conditions $[14,15]$. Therefore, the use of CFD technology is more feasible than the use of a prototype in pump design optimization and in the establishment of a preliminary forecast of pump performance.

4.1. Governing Equations and Boundary Conditions. The flow field information of pumps can be described by Navier-Stoke equations. A numerical simulation is performed to solve the following governing equations [16]:

$$
\begin{gathered}
\frac{\partial \rho}{\partial t}+\frac{\partial}{\partial x_{i}}\left(\rho u_{i}\right)=0 \\
\frac{\partial\left(\rho u_{i}\right)}{\partial t}+\frac{\partial\left(\rho u_{i} u_{j}\right)}{\partial x_{j}}=-\frac{\partial p}{\partial x_{i}}+\frac{\partial e_{i j}}{\partial x_{j}} .
\end{gathered}
$$

Fluent 6.2 was utilized in this study. The 3D unsteady flow of centrifugal pumps was calculated based on Reynoldsaveraged equations that resemble the standard renormalization group $k-\varepsilon$ turbulence model. Velocity, turbulent kinetic
TABLE 4: Head at design flow contrast of different stages (m).

\begin{tabular}{lccccc}
\hline Stage & 1st head & 2nd head & 3rd head & 4th head & Total head \\
\hline 1 & 16.93 & & & & 16.56 \\
2 & 16.89 & 16.53 & & & 32.17 \\
3 & 16.87 & 16.62 & 16.77 & & 48.87 \\
4 & 16.79 & 16.60 & 16.92 & 16.84 & 64.50 \\
\hline
\end{tabular}

energy, and eddy viscosity coefficients were provided as a first-order upwind scheme. The velocity at the inlet and free flow at the outlet were selected with boundary conditions. A solid wall was set as the no-slip condition, and a smooth wall condition was employed as the near-wall function. The convergence precision was set to $10^{-5}$.

4.2. Computational Domain. The flow domain of the submersible pump is composed of the inlet, a multistage distorting impeller, a multistage space diffuser, and an outlet. Its flow pattern is more complex than that of a single-stage pump. A rotational flow exists at the impeller inlet, but the first stage and flow in the channel are similar. Demands on computer performance would be made if all stages are considered in the calculation. Therefore, selecting only the appropriate stages is important. Four kinds of computational domains in different 
TABLE 5: Shaft power at design flow contrast of different stages (kW).

\begin{tabular}{lccccc}
\hline Stage & $\begin{array}{c}\text { 1st Shaft } \\
\text { power }\end{array}$ & $\begin{array}{c}\text { 2nd Shaft } \\
\text { power }\end{array}$ & $\begin{array}{c}\text { 3rd Shaft } \\
\text { power }\end{array}$ & $\begin{array}{c}\text { 4th Shaft } \\
\text { power }\end{array}$ & $\begin{array}{c}\text { Total shaft } \\
\text { power }\end{array}$ \\
\hline 1 & 0.853 & & & & 0.853 \\
2 & 0.855 & 0.856 & & & 1.711 \\
3 & 0.857 & 0.854 & 0.851 & & 2.562 \\
4 & 0.851 & 0.854 & 0.858 & 0.861 & 3.424 \\
\hline
\end{tabular}

TABLE 6: Efficiency at design flow contrast of different stages (\%).

\begin{tabular}{lccccc}
\hline Stage & $\begin{array}{c}\text { 1st } \\
\text { efficiency }\end{array}$ & $\begin{array}{c}\text { 2nd } \\
\text { efficiency }\end{array}$ & $\begin{array}{c}\text { 3rd } \\
\text { efficiency }\end{array}$ & $\begin{array}{c}\text { 4th } \\
\text { efficiency }\end{array}$ & $\begin{array}{c}\text { Total } \\
\text { efficiency }\end{array}$ \\
\hline 1 & 59.25 & & & & 58.38 \\
2 & 59.34 & 61.77 & & & 60.78 \\
3 & 59.47 & 61.73 & 61.79 & & 60.81 \\
4 & 59.41 & 61.82 & 61.93 & 61.87 & 60.85 \\
\hline
\end{tabular}

stages are simulated for scheme 1 . The relevant results are presented in Tables 4, 5, and 6 .

Tables 4 and 5 indicate that the head and shaft power of each stage in the pump are very similar that the two-stage values can be regarded as the corresponding values of the pump. Table 6 shows that only a few differences exist in terms of efficiency; however, many differences were noted between the first stage and other stages after the second. No changes were observed after stage two. In summary, it is appropriate to select two full-flow models to analyze pump performance. Similarly, the use of the head and shaft power of two stages as well as the efficiency of stage two to predict performance is also feasible. The computational domain composed of the inlet, the distorting impeller of two stages, the space diffusers, and the outlet is shown in Figure 2. The first pump water chamber is not shown in the figure.

4.3. Model Meshing. The quantity and quality of the grid are two important factors that affect computation accuracy and duration. The unstructured tetrahedral mesh provided by GAMBIT, the professional software for grid generation, was utilized on the entire basin. The mesh has strong adaptability. Encryption and nonequidistance were processed in the near-wall region. Five different mesh sizes were selected to determine the appropriate number of grids and to conduct a grid-independent analysis. Scheme 1 at $Q_{d}$ was regarded as an example. The calculated results are shown in Table 7.

Table 7 shows that the difference between pump head does not exceed $0.5 \%$, efficiency does not exceed $0.06 \%$, and shaft power does not exceed $0.3 \%$ when the mesh size is smaller than $2.0 \mathrm{~mm} . y^{+}$, which was utilized to examine the closest node to the surface, is not greater than 150 . Thus, the criteria of standard renormalization group $k-\varepsilon$ turbulence model calculation were met. Therefore, numerical simulation grid size of $2.0 \mathrm{~mm}$ was selected after considering computation accuracy and duration. The grid view of computational domain is shown in Figure 3.

One of the convergence plots is shown in Figure 4; this plot can be used to justify the ultimate choice of grid
TABLE 7: Results of different mesh sizes.

\begin{tabular}{lccccc}
\hline \multirow{2}{*}{ Parameter } & \multicolumn{5}{c}{ Mesh size/mm } \\
& 1.6 & 1.8 & 2.0 & 2.2 & 2.4 \\
\hline Grids/10 & 184 & 129 & 91 & 69.4 & 53.4 \\
Head/m & 32.58 & 32.33 & 32.17 & 32.03 & 31.87 \\
Efficiency $\eta / \%$ & 61.82 & 61.81 & 61.77 & 61.46 & 60.53 \\
Shaft Power $P / \mathrm{kW}$ & 1.716 & 1.706 & 1.711 & 1.694 & 1.686 \\
\hline
\end{tabular}

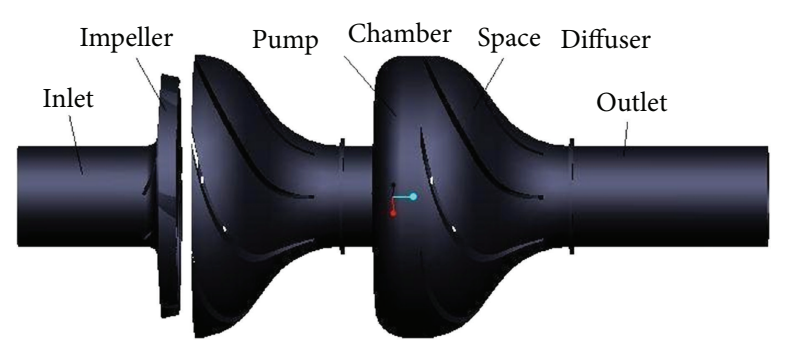

FIGURE 2: Schematic of the computational domain.

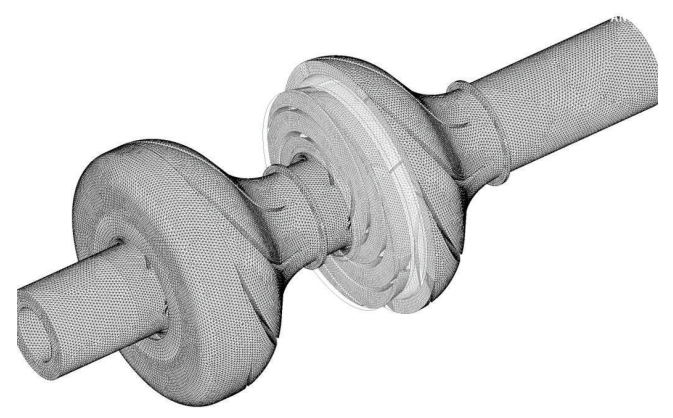

FIGURE 3: Grid view of computational domain.

for the analysis. According to Figure 4, all the convergence precisions reached $10^{-5}$, which satisfied the requirement of the calculation.

\section{Results Analysis}

Given that the shaft power of low-specific-speed centrifugal pumps increases rapidly with the enlargement of flow, the shaft power at $1.5 Q_{d}$ was selected as the monitoring value for overload judging. An electric pump works safely at the range of $0.7 Q_{d}$ to $1.3 Q_{d}$. If the shaft power at $1.5 Q_{d}$ is less than the motor power, then the pump will not experience an overload. A total of 27 numerical simulations were performed. The results of two-stage head $H$ under the rated condition, shaft power $P$ at $1.5 Q_{d}$, and efficiency $\eta$ of stage two under the rated condition are shown in Table 8.

5.1. Visual Analysis. Orthographic experimental data were analyzed. With the first column of rated point head index $H$ as an example,

$$
\begin{aligned}
k_{1 A}= & (32.17+32.81+30.25+38.08 \\
& +34.97+35.22+35.94+36.78+38.56) \\
\times & (9)^{-1}=34.977,
\end{aligned}
$$


TABLE 8: Simulation statistics.

\begin{tabular}{|c|c|c|c|}
\hline Test number & $H / \mathrm{m}$ & $\eta / \%$ & $P / \mathrm{kW}$ \\
\hline 1 & 32.17 & 61.77 & 1.711 \\
\hline 2 & 32.81 & 63.41 & 1.669 \\
\hline 3 & 30.25 & 60.45 & 1.561 \\
\hline 4 & 38.08 & 62.08 & 2.108 \\
\hline 5 & 34.97 & 61.05 & 1.983 \\
\hline 6 & 35.22 & 60.84 & 2.01 \\
\hline 7 & 35.94 & 57.41 & 2.17 \\
\hline 8 & 36.78 & 59.11 & 2.212 \\
\hline 9 & 38.56 & 61.02 & 2.242 \\
\hline 10 & 34.88 & 59.12 & 2.054 \\
\hline 11 & 35.04 & 60.10 & 2.006 \\
\hline 12 & 35.64 & 61.58 & 1.987 \\
\hline 13 & 35.80 & 61.08 & 2.064 \\
\hline 14 & 36.89 & 61.21 & 2.097 \\
\hline 15 & 35.04 & 60.12 & 2.064 \\
\hline 16 & 37.12 & 62.32 & 2.029 \\
\hline 17 & 34.50 & 59.28 & 1.989 \\
\hline 18 & 35.72 & 61.13 & 2.005 \\
\hline 19 & 38.01 & 61.73 & 2.151 \\
\hline 20 & 35.42 & 60.03 & 2.108 \\
\hline 21 & 35.36 & 60.21 & 2.092 \\
\hline 22 & 34.16 & 59.62 & 1.924 \\
\hline 23 & 33.78 & 60.64 & 1.978 \\
\hline 24 & 34.24 & 60.90 & 1.831 \\
\hline 25 & 35.89 & 60.52 & 2.080 \\
\hline 26 & 37.45 & 61.88 & 2.110 \\
\hline 27 & 35.30 & 59.59 & 2.069 \\
\hline
\end{tabular}

$H(\mathrm{~m})$ is two-stage head under $Q ; \eta(\%)$ is the efficiency of stage two under $Q ; P(\mathrm{~kW})$ is two-stage shaft power at $1.5 Q_{d}$.

$$
\begin{aligned}
k_{2 A}= & (34.88+35.04+35.64+35.80+36.89 \\
& +35.04+37.12+34.50+35.72) \\
& \times(9)^{-1}=35.627 \\
k_{3 A}= & (38.01+35.42+35.36+34.16+33.78 \\
& +34.24+35.89+37.45+35.30) \\
& \times(9)^{-1}=35.513 .
\end{aligned}
$$

Where $k_{1 A}, k_{2 A}$ and $k_{3 A}$ in the equations stand for the average efficiency values at the rated point of factor $A$. $s$, the range, is introduced to reflect the effect of various factors on the target value. A large range signifies an important factor whose change greatly affects the objective. If the range is small, then the factor has minimal influence and is usually not important. $s$ was obtained by the subtraction of the minimum from the maximum among $k_{1 A}, k_{2 A}$, and $k_{3 A}$ in each column. The calculation methods for the other nine columns were similar to the first one. The efficiency under the rated condition and shaft power at $1.5 Q_{d}$ were obtained. $k_{1}, k_{2}, k_{3}$, and $s$ of each factor are listed in Table 9.
Visual analysis indicates that the order of the importance of the index for the head is CJBDEAHFGI, and its optimal plan is $A_{2} B_{3} C_{3} B_{2} E_{1} F_{1} G_{2} H_{2} I_{2} J_{2}$. The order of importance of the index for efficiency is JDCBGHAFEI, and its optimal plan is $A_{1} B_{1} C_{1} D_{1} E_{2} F_{2} G_{2} H_{2} I_{2} J_{2}$. Similarly, the order of importance for shaft power is CBDAEIJFHG, and its optimal plan is $A_{1} B_{1} C_{1} D_{1} E_{3} F_{2} G_{2} H_{3} I_{3} J_{3}$. The integrated balance method even drawing was utilized to select the optimal program, resulting in large amounts of calculation and difficulty in establishing choices for multitarget optimization. If the matrix method introduced in this study is selected, an optimal plan can be immediately established because only three investigation indexes are calculated. The optimal program is determined by the weight value.

5.2. Weight Matrix Method. Weight matrix analysis is necessary to calculate the weight matrix of the investigation index. The weight calculation of the head was regarded as an example; high values were considered good. Thus, $K_{i j}=k_{i j}$, $T_{i}=1 / \sum_{j=1}^{m} K_{i j}, S_{i}=s_{i} / \sum_{i=1}^{l} s_{i}$, and $\omega=M \cdot T \cdot S$.

In general, higher efficiency is also considered good. Hence, $K_{i j}=k_{i j}, T_{i}=1 / \sum_{j=1}^{m} K_{i j}, S_{i}=s_{i} / \sum_{i=1}^{l} s_{i}$. Furthermore, low shaft power is desirable. Thus, $K_{i j}=$ $1 / k_{i j}, T_{i}=1 / \sum_{j=1}^{m} K_{i j}$, and $S_{i}=s_{i} / \sum_{i=1}^{l} s_{i}$. The general weight matrix in (14) is obtained after substituting the above calculations into (4) as follows:

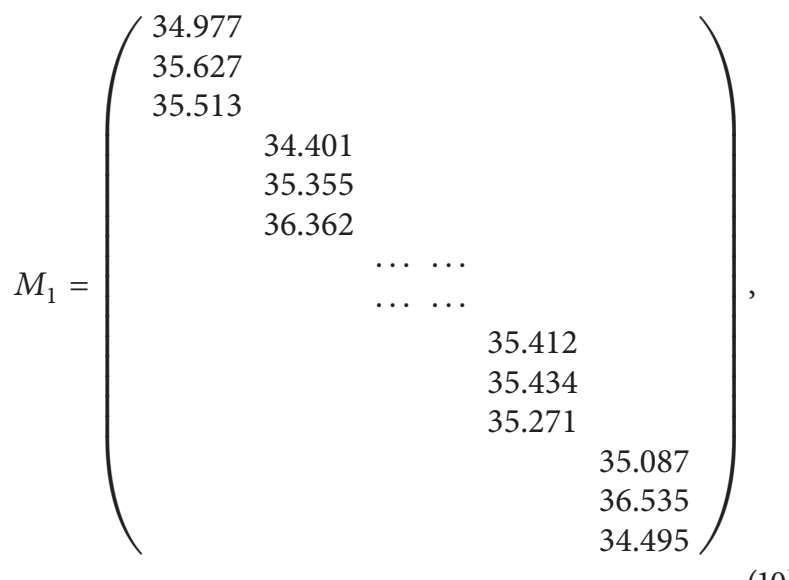

$$
T_{1}=\left(\begin{array}{lll}
0.00942 & & \\
& \cdots & \\
& & 0.00942
\end{array}\right)
$$

$$
S_{1}=\left(\begin{array}{c}
0.0620 \\
0.1870 \\
0.2442 \\
0.1353 \\
0.0711 \\
0.0211 \\
0.01587 \\
0.05342 \\
0.01559 \\
0.1945
\end{array}\right),
$$$$
\omega_{1}=M_{1} \cdot T_{1} \cdot S_{1} \text {, }
$$ 
TABLE 9: Range analysis of orthogonal test data.

\begin{tabular}{cccccccccccc}
\hline Index & $A$ & $B$ & $C$ & $D$ & $E$ & $F$ & $G$ & $H$ & $I$ \\
\hline$H / \mathrm{m}$ & & & & & & & & & & \\
$k_{1}$ & 34.977 & 34.401 & 33.862 & 34.625 & 35.784 & 35.473 & 35.281 & 35.366 & 35.412 & 35.087 \\
$k_{2}$ & 35.627 & 35.355 & 35.831 & 36.045 & 35.295 & 35.252 & 35.447 & 35.656 & 35.434 & 36.535 \\
$k_{3}$ & 35.513 & 36.362 & 36.424 & 35.447 & 35.038 & 35.393 & 35.389 & 35.096 & 35.271 & 34.495 \\
$s$ & 0.650 & 1.961 & 2.562 & 1.420 & 0.746 & 0.221 & 0.167 & 0.560 & 0.163 & 2.040 \\
$\eta / \%$ & & & & & & & & & \\
$k_{1}$ & 60.795 & 60.935 & 61.059 & 61.116 & 60.630 & 60.739 & 60.285 & 60.530 & 60.678 & 60.600 \\
$k_{2}$ & 60.661 & 60.838 & 60.754 & 60.965 & 60.747 & 60.755 & 60.920 & 60.897 & 60.713 \\
$k_{3}$ & 60.571 & 60.254 & 60.214 & 59.946 & 60.650 & 60.532 & 60.822 & 60.601 & 60.636 \\
$s$ & 0.224 & 0.681 & 0.844 & 1.170 & 0.117 & 0.223 & 0.635 & 0.367 & 0.077 & 2.163 \\
$P / \mathrm{kW}$ & & & & & & & & & \\
$k_{1}$ & 1.963 & 1.927 & 1.855 & 1.936 & 2.033 & 2.027 & 2.009 & 2.015 & 2.017 \\
$k_{2}$ & 2.033 & 2.007 & 2.045 & 2.053 & 2.017 & 2.000 & 2.008 & 2.022 & 2.031 & 2.018 \\
$k_{3}$ & 2.038 & 2.101 & 2.134 & 2.045 & 1.985 & 2.007 & 2.017 & 1.998 & 1.987 & 1.992 \\
$s$ & 0.075 & 0.174 & 0.279 & 0.116 & 0.048 & 0.027 & 0.009 & 0.024 & 0.043 & 0.033 \\
\hline
\end{tabular}

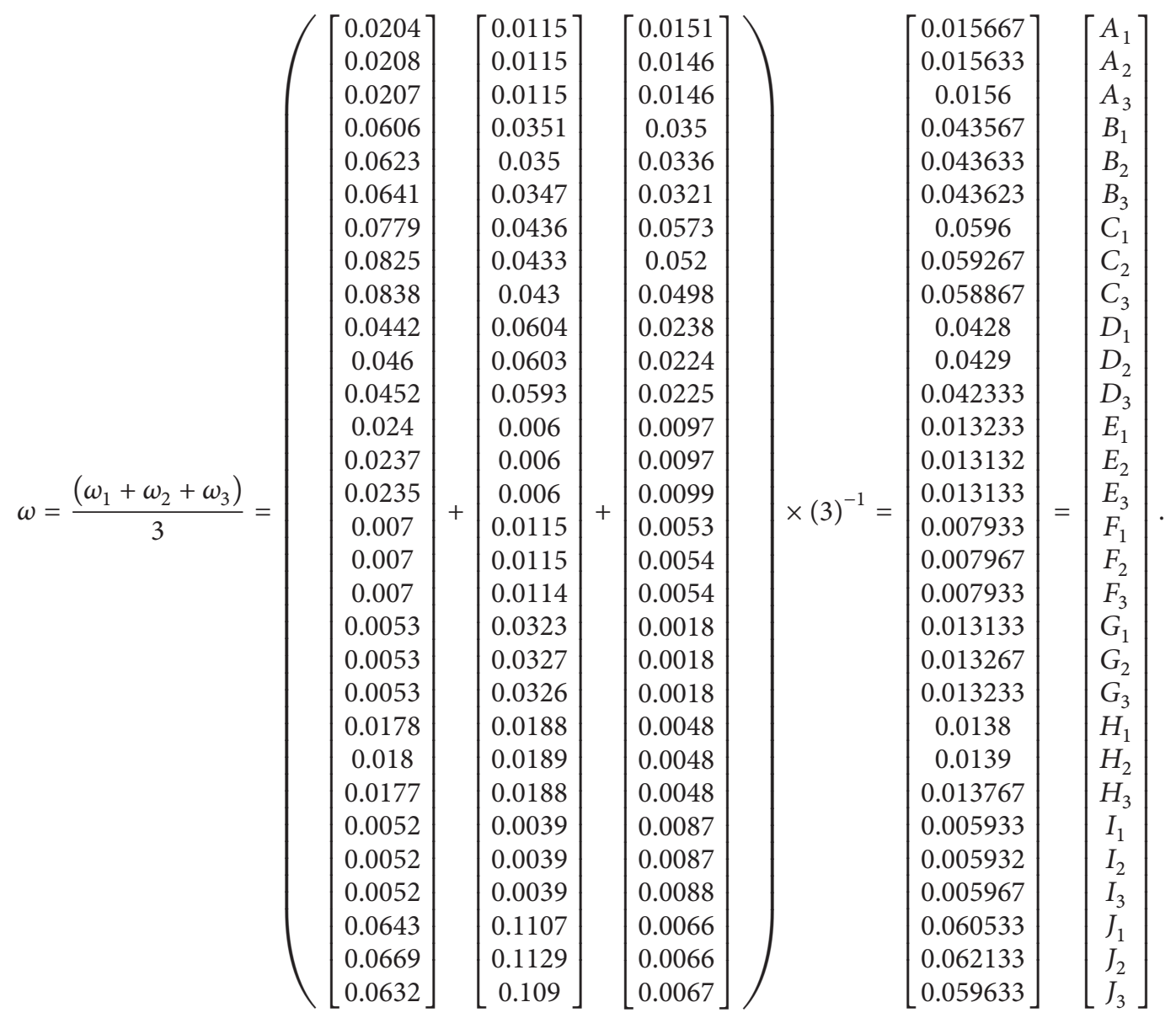




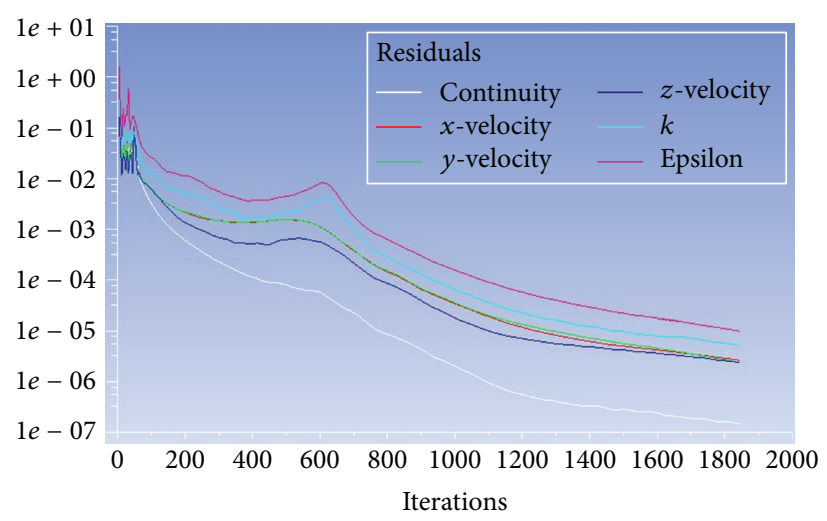

FIgure 4: Convergence plots of the CFD calculation.

The calculations provide the weight of the result of each factor with three levels. Hence, the optimal plan for orthographic experiments is determined quickly. The optimal plan is $A_{1} B_{2} C_{2} D_{2} E_{1} F_{2} G_{2} H_{2} I_{3} J_{2}$, and the importance order of the index is JDCGHEABFI.

\section{Experimental Verification}

The optimal model, $J_{2} D_{2} C_{2} G_{2} H_{2} E_{1} A_{1} B_{2} F_{2} I_{3}$, was prototyped for the experiment to test the optimized programs. The geometric parameters of the hydraulic model were as follows: width of the blade outlet $b_{2}=9 \mathrm{~mm}$, inclination of back cover board $\alpha_{2}=89^{\circ}$, inlet attack angel $\Delta \beta_{1}=10^{\circ}$, inlet angle in axial plane $\gamma_{3}=10^{\circ}$, blade outlet angle $\beta_{2}=15^{\circ}$, blades number $z=7$, outer diameter of back cover $d_{2 \min }=$ $122 \mathrm{~mm}$, blade thickness $S_{2}=2 \mathrm{~mm}$, inlet angle of guide vane $\beta_{3}=20^{\circ}$, and axial length of the guide vane $\gamma_{3}=10^{\circ}$. The test was performed in accordance with the national standards GB/T 12785-2002 of China. Figure 5 presents the results of the experiment and the entire flow field simulation.

Comparison of the simulation and experimental results indicates that the simulated head and efficiency are slightly higher than those of the experiment; however, the simulated power is slightly lower. Error analysis indicates the following: (1) the numerical simulation did not consider the loss of leakage at the oral ring and between stages; (2) small differences exist because of limitations imposed by casting accuracy, casting errors, casting model (particularly the impeller), and simulated model; these differences can cause disparity between experimental and simulated values. However, the error is less than 5\%, and both experimental and simulated values exhibit similar trends when the flow changes. Hence, the simulation methods employed in this study can meet the demand of practical usage in programs for design optimization.

The efficiency of QS10-68/4 based on the national standard of China is $51 \%$. After optimization, the pump's rated head is $68.9 \mathrm{~m}$ and rated efficiency is $58.61 \%$, which is approximately $7 \%$ higher than the national standard. The maximum shaft power is $3.83 \mathrm{~kW}$. The performance of nonoverload also meets the demand, which is $4 \mathrm{~kW}$. All of the above data indicate that optimization was successful.

\section{Conclusions}

The influence of 10 factors and three levels on the head, efficiency, and shaft power was analyzed by conducting 27 orthographic experiments through numerical simulation. Weight matrix analysis was then performed to determine the optimal model. The principal conclusions drawn from this research are as follows.

(1) The simulation results indicate that the inlet setting angle of the guide vane $\beta_{3}$ significantly affects the efficiency and that the outlet angle of blades $\beta_{2}$ has a significant effect on the head and shaft power.

(2) The importance order of the index for the head is $\beta_{2} \beta_{3} b_{2} z S_{2} d_{2 \min } \Delta \beta_{1} \alpha_{2} d_{1} \gamma_{3}$, and the geometric parameters of optimal plan are as follows: $d_{2 \min }=124 \mathrm{~mm}$, $b_{2}=10 \mathrm{~mm}, \beta_{2}=20^{\circ}, z=7, S_{2}=2 \mathrm{~mm}, \alpha_{2}=$ $88^{\circ}, d_{1}=36 \mathrm{~mm}, \Delta \beta_{1}=10^{\circ}, \gamma_{3}=5^{\circ}$, and $\beta_{3}=$ $20^{\circ}$. The importance order of the index for efficiency is $\beta_{3} z \beta_{2} b_{2} d_{1} \Delta \beta_{1} d_{2 \min } \alpha_{2} S_{2} \gamma_{3}$, and the geometric parameters of optimal plan are as follows: $d_{2 \min }=$ $122 \mathrm{~mm}, b_{2}=8 \mathrm{~mm}, \beta_{2}=10^{\circ}, z=6, S_{2}=3 \mathrm{~mm}$, $\alpha_{2}=89^{\circ}, d_{1}=36 \mathrm{~mm}, \Delta \beta_{1}=10^{\circ}, \gamma_{3}=5^{\circ}$, and $\beta_{3}=20^{\circ}$. For shaft power, the importance order of the index is $\beta_{2} b_{2} z d_{2 \min } S_{2} \gamma_{3} \beta_{3} \alpha_{2} \Delta \beta_{1} d_{1}$, and the geometric parameters of optimal plan are as follows: $d_{2 \min }=$ $122 \mathrm{~mm}, b_{2}=8 \mathrm{~mm}, \beta_{2}=10^{\circ}, z=6, S_{2}=4 \mathrm{~mm}$, $\alpha_{2}=89^{\circ}, d_{1}=36 \mathrm{~mm}, \Delta \beta_{1}=15^{\circ}, \gamma_{3}=10^{\circ}$, and $\beta_{3}=25^{\circ}$.

(3) By performing weight matrix analysis, optimal model was obtained based on the influence of factors and levels on the head, efficiency, and shaft power. The geometric parameters of the hydraulic model were as follows: $b_{2}=9 \mathrm{~mm}, \alpha_{2}=89^{\circ}, \Delta \beta_{1}=10^{\circ}, \gamma_{3}=10^{\circ}$, $\beta_{2}=15^{\circ}, z=7, d_{2 \min }=122 \mathrm{~mm}, S_{2}=2 \mathrm{~mm}$, $\beta_{3}=20^{\circ}$, and $\gamma_{3}=10^{\circ}$. The performance of the prototype in the test indicates that efficiency at the rated point is $7 \%$ higher than the national standard 


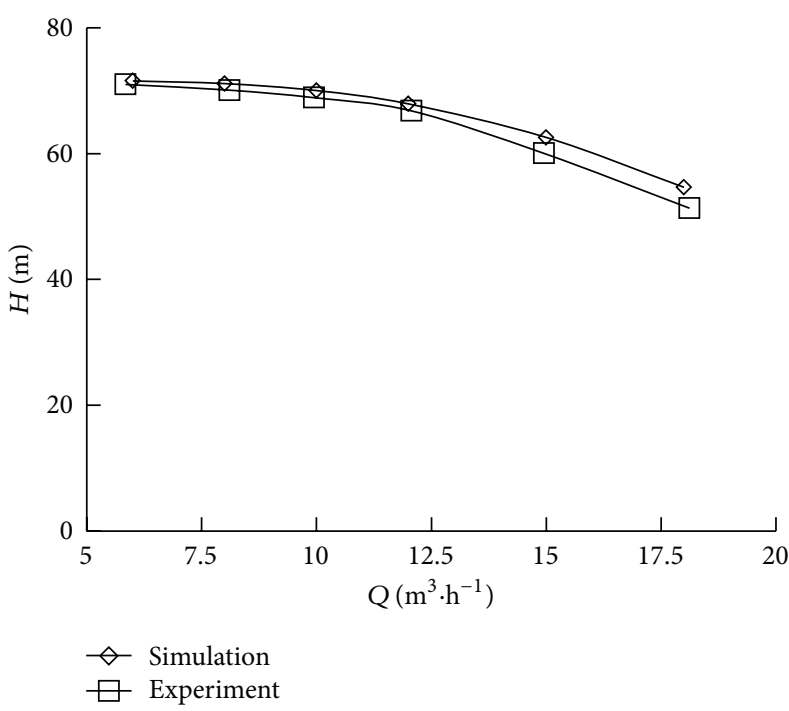

(a) Head flow curve

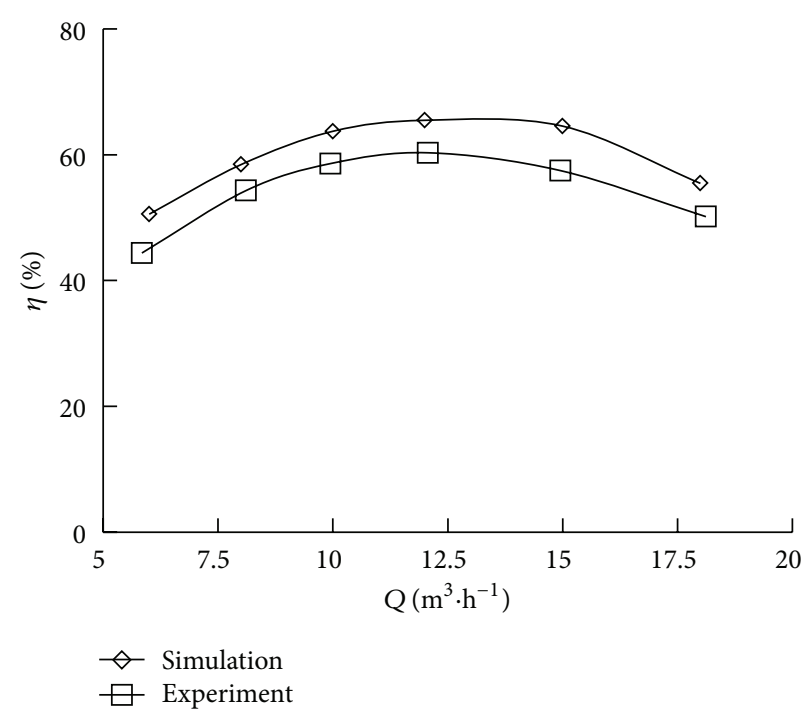

(b) Efficiency flow curve

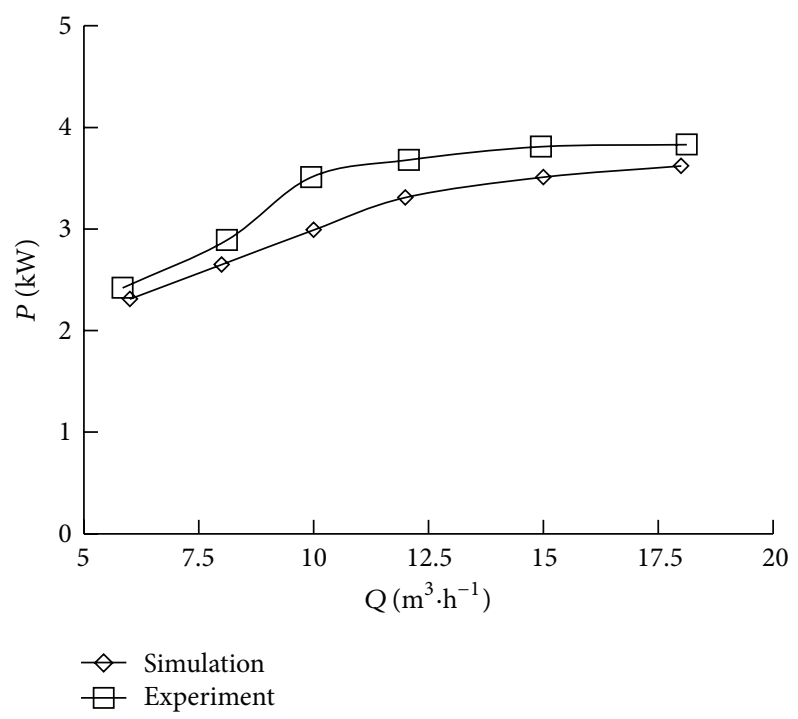

(c) Shaft power flow curve

Figure 5: Contrast diagram between the simulation and experiment.

of China. This result verifies the potential use of the orthographic design combined matrix analysis with numerical simulation in pump optimization.

(4) The changes in the head, efficiency, and shaft power determined from the simulation are in accordance with the test results. The error between numerical simulation and test result is less than $5 \%$ in the rated flow, which further verifies the possibility of forecasting the performance of multistage pumps through numerical simulation.

\section{Acknowledgments}

This work was financially supported by the State Key Program of National Natural Science of China (Grant no. 51239005) and the National Science \& Technology Pillar Program
(Grant no. 2011BAF14B04) of China. This research was also sponsored by the Research and Innovation Project for College Graduates of Jiangsu Province (CXZZ12_0679).

\section{References}

[1] F. G. Johann, Centrifugal Pumps, Springer, New York, NY, USA, 2008.

[2] Y. Shouqi, C. Wuling, and L. Shiying, "Theory and design method of non-overload centrifugal pumps," Chinese Journal of Mechanical Engineering, vol. 5, no. 4, pp. 252-260, 1992.

[3] Z. peifang, Y. Shouqi, and X. Jianqiang, "Present status and development trends of low-specific-speed centrifugal pumps," Drainge and Irrigation Machinery, vol. 21, no. 6, pp. 42-45, 2003.

[4] M. A. Moreno, J. I. Córcoles, D. A. Moraleda, A. Martinez, and J. M. Tarjuelo, "Optimization of underground water pumping," 
Journal of Irrigation and Drainage Engineering, vol. 136, no. 6, pp. 414-420, 2010.

[5] M. Moradi-Jalal, S. I. Rodin, and M. A. Marino, "Use of genetic algorithm in optimization of irrigation pumping stations," Journal of Irrigation and Drainage Engineering, vol. 130, no. 5, pp. 357-365, 2004.

[6] A. S. Hedayat, N. J. A. Sloane, and J. Stufken, Orthogonal Arrays, Theory and Applications, Springer, New York, NY, USA, 1999.

[7] Z. Lixue, S. Yaozhen, L. Qingsheng et al., "Mathematic model of seed peeling efficiency with critical structure parameters based on orthogonal multinomial tests," Transactions of the CSAE, vol. 19, no. 5, pp. 22-26, 2003.

[8] Y. Shouqi, Z. Jinfeng, Y. Jianping et al., "Orthogonal experimental study effect of main geometry factors of splitter blades on pump performance," And Irrigation Machinery, vol. 26, no. 2, pp. 1-5, 2008.

[9] H. Wang, W. Shi, W. Lu, L. Zhou, and C. Wang, "Optimization design of deep well pump based on latin square test," Transactions of the Chinese Society of Agricultural Machinery, vol. 41, no. 5, pp. 56-63, 2010 (Chinese).

[10] Y. Shen, S. Yuan, W. Lu, J. Zhang, and J. Yuan, "Orthogonal test design method based on numerical simulation for nonoverload centrifugal pump with complex impeller," Transactions of the Chinese Society of Agricultural Machinery, vol. 41, no. 9, pp. 22-26, 2010 (Chinese).

[11] L. Zhou, W. Shi, W. Lu, R. Xu, and C. Wang, "Orthogonal test and optimization design of submersible pump guide vanes," Journal of Drainage and Irrigation Machinery Engineering, vol. 29, no. 4, pp. 312-342, 2011.

[12] W. Shi, T. Jiang, W. Cao, W. Lu, D. Zhang, and B. Chen, "Optimal design and experiment on a high-head non-overload submersible sewage pump," Transactions of the Chinese Society of Agricultural Engineering, vol. 27, no. 5, pp. 151-155, 2011.

[13] S. N. Shukla and J. T. Kshirsagar, "Numerical experiments on a centrifugal pump," in Proceedings of the 2002 ASME Joint U.S.European Fluids Engineering Division Conference (FEDSM '02), pp. 709-720, July 2002.

[14] R. K. Byskov, C. B. Jacobsen, and N. Pedersen, "Flow in a centrifugal pump impeller at design and off-design conditions. Part II: large eddy simulations," Journal of Fluids Engineering, vol. 125, no. 1, pp. 73-83, 2003.

[15] R. Spence and J. Amaral-Teixeira, "A CFD parametric study of geometrical variations on the pressure pulsations and performance characteristics of a centrifugal pump," Computers and Fluids, vol. 38, no. 6, pp. 1243-1257, 2009.

[16] J. H. Ferziger, Computational Methods for Fluid Dynamics, Springer, Berlin, Germany, 2002. 


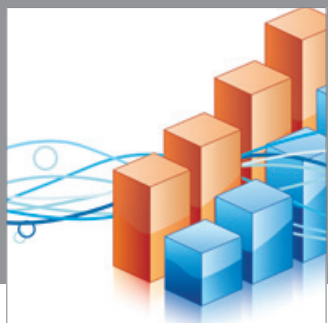

Advances in

Operations Research

mansans

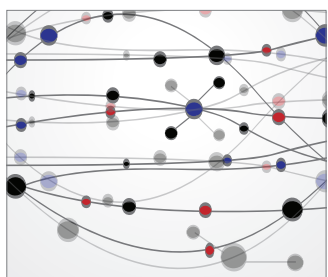

The Scientific World Journal
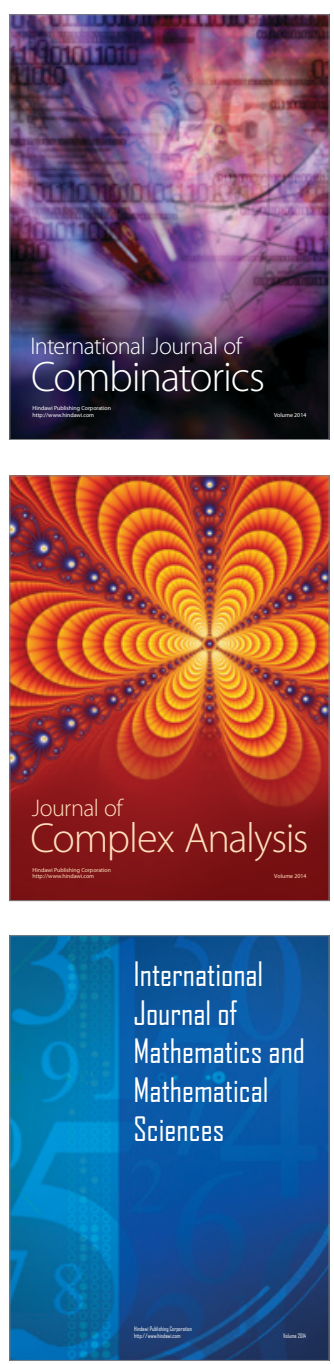
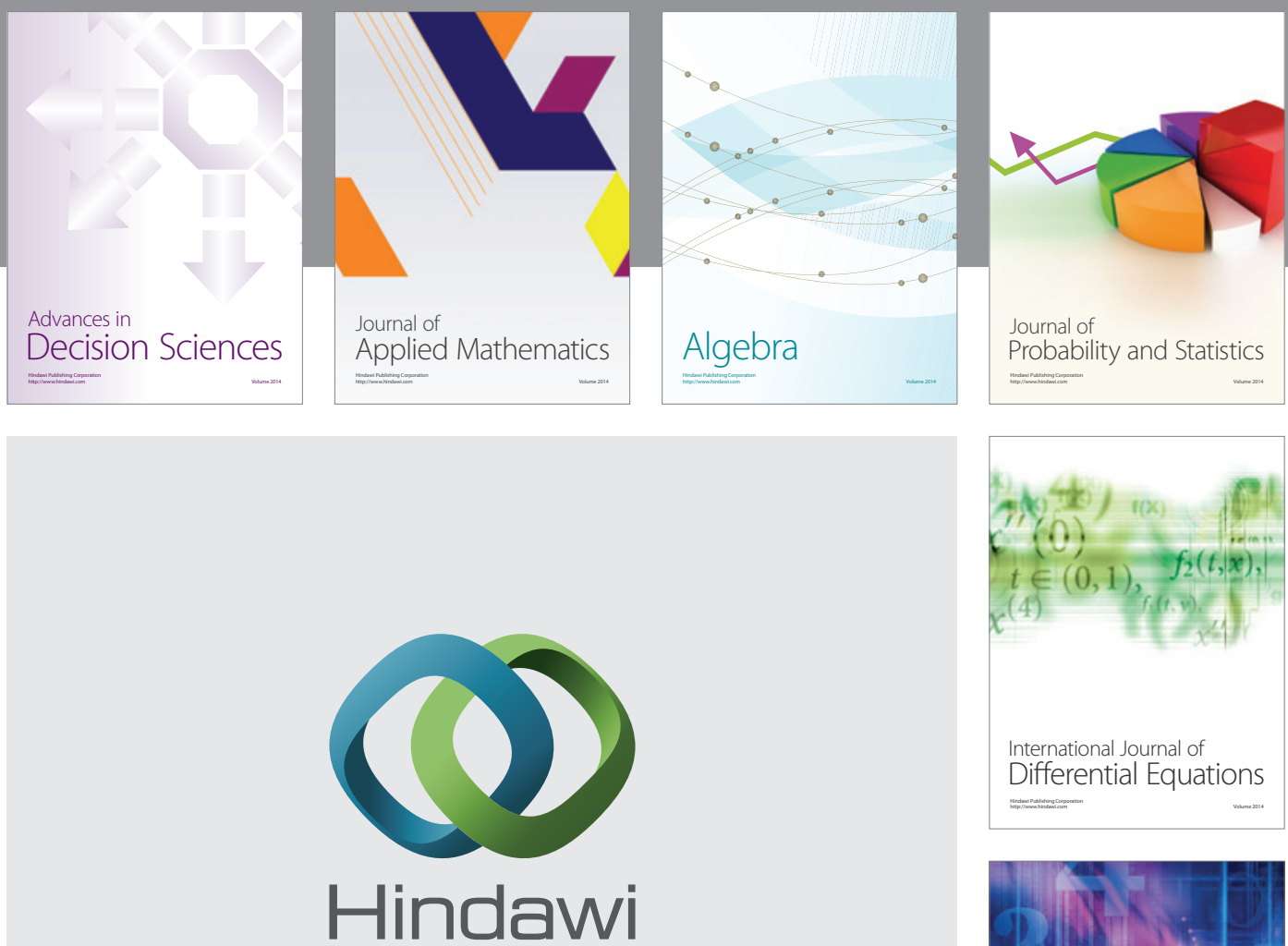

Submit your manuscripts at http://www.hindawi.com
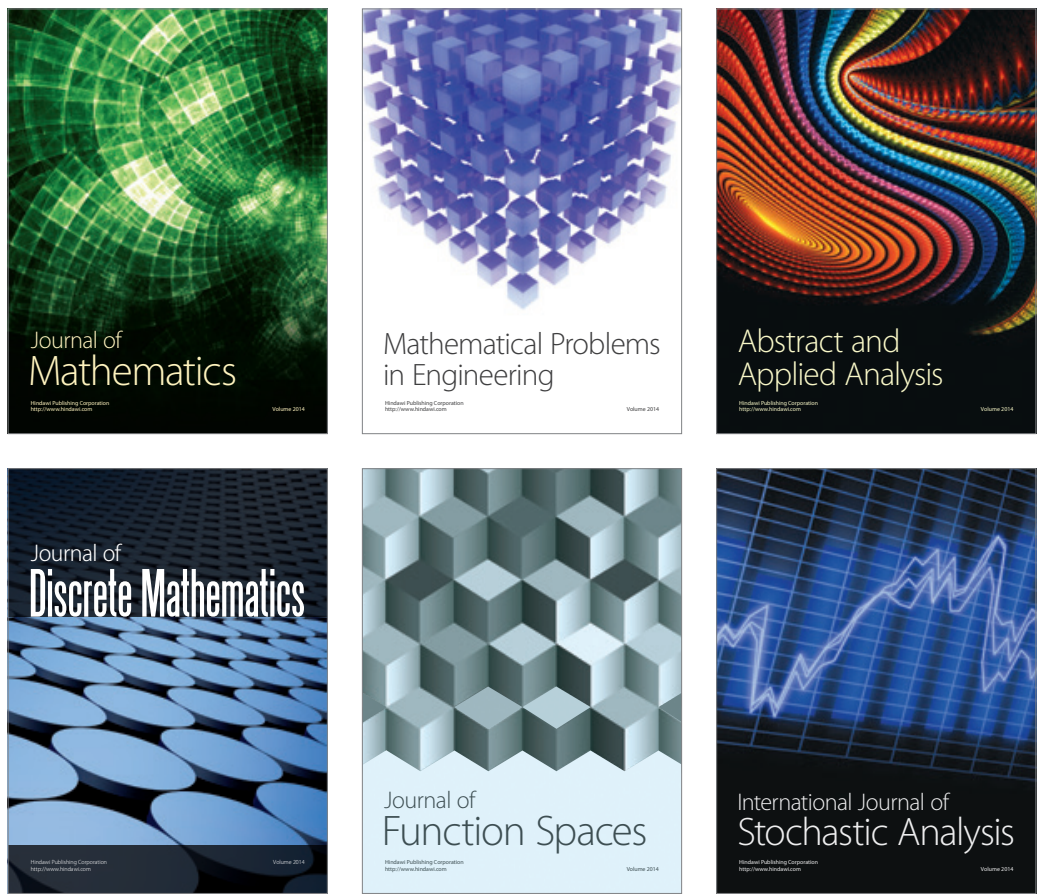

Journal of

Function Spaces

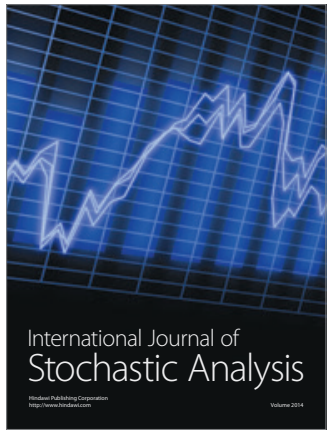

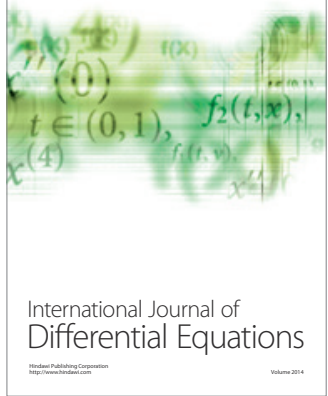
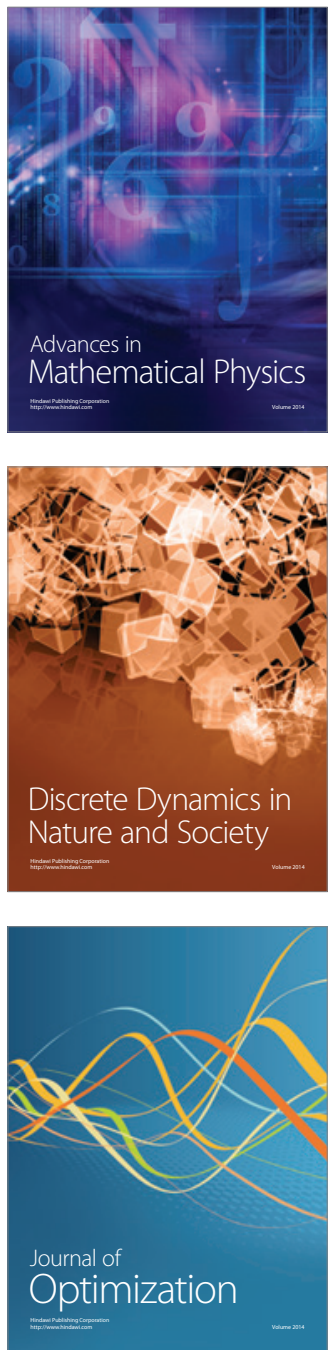